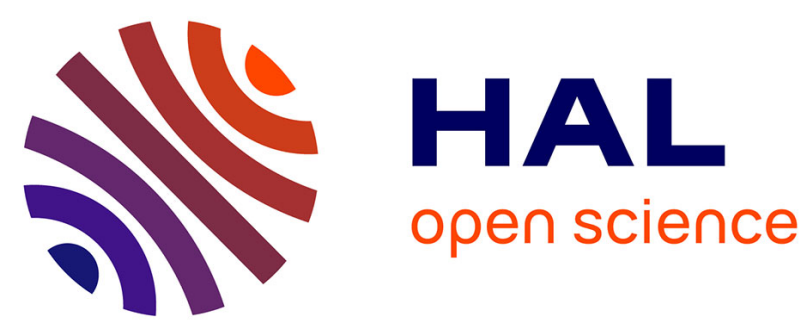

\title{
STUDY OF THE RECOMBINATION GASDYNAMIC LASERS ON ELECTRONIC TRANSITIONS (VISIBLE GDL). CURRENT STATUS
}

V. Kochelap, I. Izmailov, L. Kernazhitsky, V. Naumov

\section{- To cite this version:}

V. Kochelap, I. Izmailov, L. Kernazhitsky, V. Naumov. STUDY OF THE RECOMBINATION GASDYNAMIC LASERS ON ELECTRONIC TRANSITIONS (VISIBLE GDL). CURRENT STATUS. Journal de Physique IV Proceedings, 1991, 01 (C7), pp.C7-637-C7-642. 10.1051/jp4:19917171 . jpa00250849

\section{HAL Id: jpa-00250849 https://hal.science/jpa-00250849}

Submitted on 1 Jan 1991

HAL is a multi-disciplinary open access archive for the deposit and dissemination of scientific research documents, whether they are published or not. The documents may come from teaching and research institutions in France or abroad, or from public or private research centers.
L'archive ouverte pluridisciplinaire HAL, est destinée au dépôt et à la diffusion de documents scientifiques de niveau recherche, publiés ou non, émanant des établissements d'enseignement et de recherche français ou étrangers, des laboratoires publics ou privés. 


\title{
STUDY OF THE RECOMBINATION GASDYNAMIC LASERS ON ELECTRONIC TRANSITIONS (VISIBLE GDL). CURRENT STATUS
}

\author{
V.A. KOCHELAP, I.A. IZMAILOV, L.A. KERNAZHITSKY and V.V. NAUMOV \\ Ukrainian Academy of Science, Institute of Semiconductors, Department of Theoretical Physics, \\ Advanced Laser Research Group, 45 Prospect Nauka, Kiev 252650, USSR
}

\begin{abstract}
In report current theoretical and experimental studies on development of a new molecular electronic transition gas flow lasers on the base of $S+S$, $C l+C l$, Br+Er radiative recombination reactions are revued.
\end{abstract}

\section{1. - Irtroduction.}

High power short wavelenght lasers on electronic transitions CETy are extremely attractive for many scientiric and industrial application. Eut all existed now visible and UV gas lasers operate in pulsed regime Crare gas-galide excimers, halogens and others. As known the convective-filow techniques can be used to provide nore efficient methods for achieving a continuous wave CCW 1 aser action. However, there is only one cW chemical oxygen-iodine laser operating on atomic ET of iodine in near IR spectrum [1]. CW lasers on molecular ET in visible spectrum have not been demonstrated yet. In the case of success, we expect a discovery of new generation of powerful and efficient gas flow lasers CGFL) [2-3].

2. General Conception. Background.

In prinoiple, from an operational viewpoint a visible GFL is similar to a wel1-devel oped CW HF, DF, CO- or CO-GDLS operating on vibration rotation transitions CVFT in mid IR spectrum. Eut to extend the idea of GFL from IR to visible region cfrom VRT to ET, some physical and technical nontrivial problems should be overcome.

The eseneral conception about the specific of ET GFL may be given without going into particulars of laser action [e]. One of the main difficulties is to produce a good intensive electronilally excited states CEES excitation, the other is to provide the conditions for population inversion during EES relaxation in gas flow. The integration of these hard-combined requiremerts imposes the strong limitations on the properties ard parameters of active medium ard dictates the choice of methods for 1 aser pumping and operational regimes.

In Table 1 comparisional characteristics for different types of 1 aser transitions are presented: pumping rate $R^{*}$ required for a light gain a $10^{-3} \mathrm{~cm}$, specific power of spontaneous emission w under the same conditions and stimulated emission oross-section o ar preserted As can be seen, for a change-over from IR to visible lasing the rate of pumping should be increased by a factor of $10^{3}$ or more. On the other harid, a molecular ET has a broad bandwidth, that leads to small cross-section of stimulated emission cin comparision with the atomic ET, thus 1 arge EES density necessitates for reasonablo gain. 
Table 1

\begin{tabular}{|c|c|c|c|c|}
\hline Value & $\begin{array}{l}\text { Molecular } \\
\text { VRT }\end{array}$ & $\begin{array}{l}\text { Atomic ET } \\
\text { (bound }\end{array}$ & $\begin{array}{l}\text { Molecular ET } \\
\text { (bound) }\end{array}$ & $\begin{array}{l}\text { Molecular ET } \\
\text { Cconti nuum }\end{array}$ \\
\hline $\begin{array}{l}\lambda, \mu \mathrm{m} \\
, / \mathrm{cm}^{3} s \\
W / \mathrm{cm}^{3} \\
\mathrm{~cm}^{2}\end{array}$ & $\begin{array}{c}2.7 .0^{12} \cdot 9 \\
10^{-5} \\
10^{-15}\end{array}$ & $\begin{array}{c}5 \times 10^{0.5} \\
10^{-3} \\
5 \times 10^{-14}\end{array}$ & $\begin{array}{c}10^{0.5} \\
5 \\
10^{-17}\end{array}$ & $\begin{array}{l}10^{22.5 \pm 0.05} \\
60 \\
10^{-18}\end{array}$ \\
\hline
\end{tabular}

In practice, these conclusions result in the high pressure actlve medium necessary to use. Therefore, the commonly used reactive flow mixing methods for visible GFL pumping could not be useful so must bring some new problems. In addition, quite a number of further 1 mportant conditions should be borne in mind creaction stability, inversion range, medium optical homogenelty, etc.J for reaching a laser effect. Still one difficulty is a lock of rellable kinetic and spectral data CEES excitation, quenching and relaxation rates, radiative 1 ifetimes and absorption cross-sections, etc. 3 crucial to the selection of suitable laser molecules and chemical reactions. All this gained the impression that the visible GFLs development would require major efforts than for conventional IR GDLs.

Radiative recombination of atoms and free radicals in gases presents an important class of highly exoenergic chemilumunescence reactions. It attracts our attention potentially as most perspective way to create a thermaliy cor chemicaliy pumped visible recombination GDL (RGDL). Unlike usual IR GDLs on VRT, RGDLs can use EES with excitation energy up to $4 \mathrm{eV}$. In contrast with relatively small fraction of vibrational energy, the energy that can be stored in the breaking chemical bonds under molecular dissociation and partitioned into EES under atomic recombination amounts to $60 \%$ If it could produce the inversion with a quantum efficiency of $50 \%$, one could create the laser with an efficiency up to $30 \%$.

The simple scheme of recombination pumping mechanisms 1 s

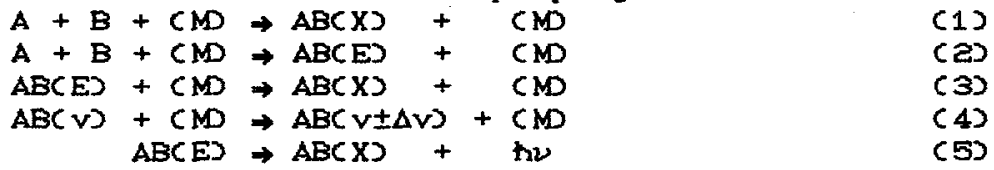

Here, $A$ and $B$ are recombining atoms, process (1) is a recombination into the ground $E S$ of product molecules $A B$, (Z) - recombination into EES, (3) - collisional quenching of EES, (4) - vibrational relaxation of ES and (S) - radiative ET to generate the laser output. (1) and ( 3 ) compose a "dark" channal of recombination, (2) and (5) determine a "light" channal. The reactive branching ratio into EES can be up to $0.25 . .0 .75$.

This kinetic mechanisms can provide the high rate of EES production and large enough quantum yield. The recombination reaction is a three-body process, its rate is $R=k r$ [A] [B] [M]. A usual order of magnitude of reaction constant is $k=1 \times 10^{-93} \mathrm{~cm}^{\delta} / \mathrm{s}$ Cover range of temperatures $T \leq 1000 \mathrm{~K}$. Therefore, tor reach a required value of EES pumping rate $R^{*}=1 \times 10^{21} \mathrm{~cm}^{3}$ s the concentration of atoms should be as high as $[A]=[B]=1 \times 10^{18} / \mathrm{cm}^{3}$ cdilution $\left.[\mathrm{M}]:[\mathrm{AB}]=1: 5\right)$. It means the process should be proceeded at strong chemical nonequilibrium conditions to shift toward to EES production. To do it, the characteristic time of gas flow, $t,=1 /$ (dlnt $/ d t)$, should be much less than the recombination time, gas $t=1 K$ [A][M].

The case of achieving a population ${ }^{\circ}$ inversion varies with the parameters of EES and presents a complex trade-off between gain and loss mechanisms. For most suitable lasants due to a shift of EES potemtial energy curve relative to the ground $E S$ and because of 
favourable Frank-Condon factors, a partial population inversion should be created between the low $v$-levels of the lasing EES and the high $v-1$ evels of the ground ES essentially unpopulated at low operational temperatures. This occurs necessarily when VT-relaxation is more rapid ther EES-desactivation.

The bandwith of radiative ET depends on both the emitting EES and the ground ES potential energy curves, as well as the distribution of excited states among the various vibrational and rotational states of laser levels. Most molecules also have metastable EEs that lie close and sometimes below the upper laser level. These EES act as energy reservoirs for excitation that can funnel through the radiative EES.

To our knowledge we have a sufficlent background for the GDLs : a) there are specific diatomic molecules as a potential lasants and favourable gas-phase chemiluminescence processes going by scheme (1)-C5) for which kinetics, spectrum and other properties are well known Chalogens, interhalides, sulfur and other candidates?;

b) high degree of dissociation of this molecules is reached at practicable temperatures of the plenum gas: for the $\mathrm{Br}$ with dissociation energy $D \simeq 2 \mathrm{eV}$ the characteristic temperature is ${ }^{2} \mathrm{~T}_{\mathrm{d}}=2000 \mathrm{~K}$, for $\mathrm{Cl} \mathrm{I}^{-}$ $D \simeq 2,5 \mathrm{eV}, T_{d}=3000 \mathrm{~K}$, for $S_{2}-D \simeq 4 \mathrm{eV}, T_{d}=4000{ }^{d} K_{;}$that allows tz produce a high atom densities in the plenum chamber under heating by electrical arc, shock waves, explosion or combustion Cthere are some fuels can contain the necessary species and can supply the necessary temperature and pressure regimes);

c) dissociated gas can be cooled to temperatures below $1000 \mathrm{~K}$ fast enough in the supersonic nozzle flow during the time $t$ as $\simeq 10^{-6} \mathrm{~s} \leq$ $t \simeq \simeq 10^{-4} s$ to initiate a EES recombination pumping ${ }^{2}$ followed by pôpิ̂ิlation inversion; the supersonic flow (Mach number $\sim 3 . .5$ ) also provides to rapid remove a waste species and heat release following laser action; the presence of diluent in gas composition promotes to extend the inversion zone along the cavity flow up to a few tens $\mathrm{cm}$.

The typical conditions selected in view of theory predictions [2] for the RGDLs demonstration are presented in Table 2 .

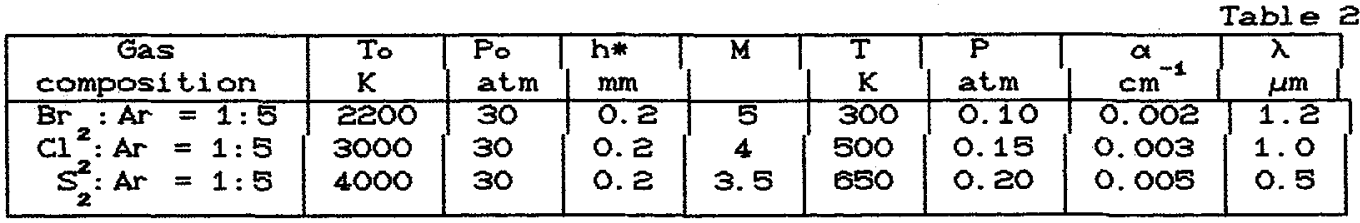

\section{3. -Results of Pre-Laser Studies. Critical Review.}

Because a complexity of RGDL's development, it takes the special strategy of research: first of all, to carry out the pre-iaser gasdynamic photochemical studies and only then to work out the RGDL-like machine. The elements of such strategy have been tested by our Advanced Laser Research Group in Kiev $[2,4-7]$.

The method of heating-fast cooling in supersonic flow conditions was realized for $C_{1}(B-X)$ EES recombination pumping and population inversion formation in the shock tube gasdymamic pre-laser experiments [4-6]. The optical (shadow technique and interferometrys and spectroscopy cemissivity and absorptivity techniques flow vizualization in complex with numerical flow modeling were used. Extremely intensive, up to $0.1 \mathrm{w} / \mathrm{cm}^{3}$. spontaneous emission in near IR with quantum yield up to 0.001 photon/molecule was observed. Using the calibrated multichanal spectroscopic measurements $c \lambda=325, \lambda=441.6$, . $\lambda_{i}=1008 \pm 8 \mathrm{~nm}$ in various diagnostic sections $\mathrm{h} / \mathrm{h}$ of tested nozzles, pópulation of ground $E S[C] C X)]$ and vibrational temperature $T$ have been defined from the gas absorbtivity A, and population of optlcally active EES $\left[\mathrm{Cl}_{2}(B)\right]$ - from the gas emissi $\lambda_{i t y} I_{\lambda}$. Radiative lifetime 
$\left.T C B, V^{\prime}=0\right)=50$ hs has been found from the preliminary measurements of inin shock waves. The typical conditions and results of experiments are summarized in Table 3 .

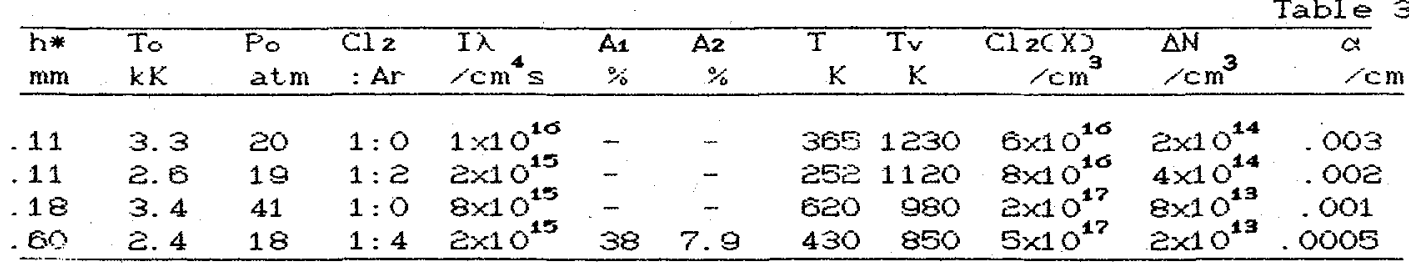

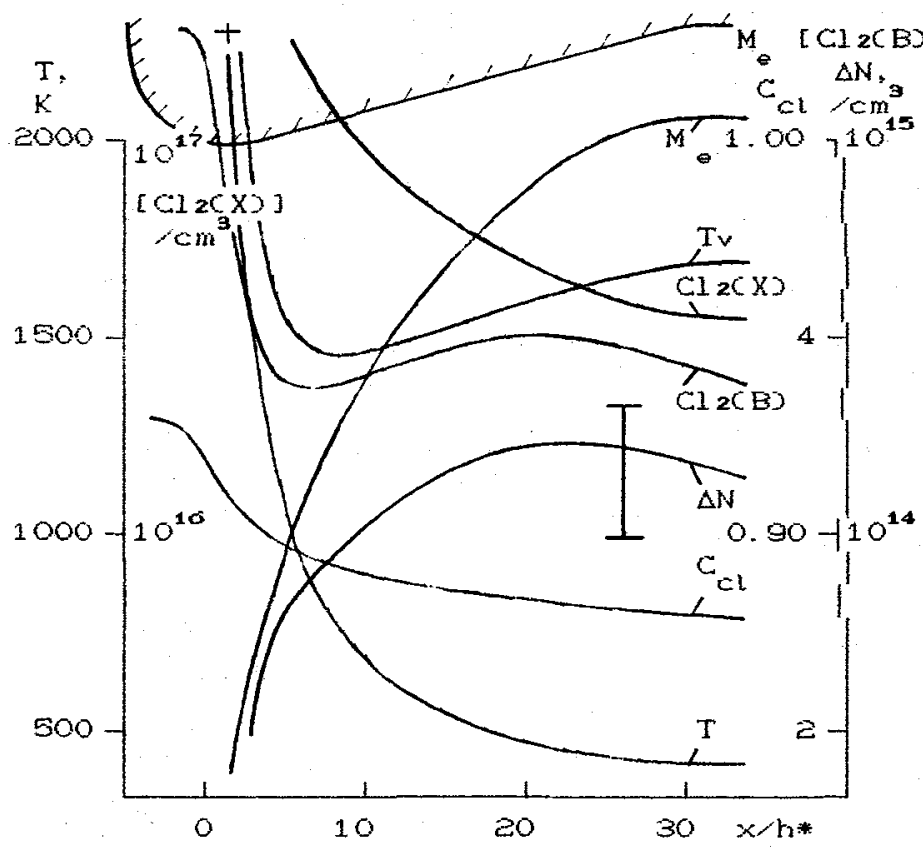

Fig. 1. Evolution of recombining gas flow parameters and population inversion formation versus distance $x / h *$ in nozzle $h *=0.18$ mm, $\theta^{\circ}=30^{\circ}$ for plenum conditior fo $_{0}=3530 \mathrm{~K}$. Fo=17 atm, pure chlorine. I - observation.
By a computational modeling, the set of important characteristics of $\mathrm{Cl}(\mathrm{B}-\mathrm{X}) \mathrm{kine-}$ tics was détermined: recombination efficiency and its branching ratio, rates of EES and virational relaxation, etc. Example of numerical simulation of nozzle flow parameters is shown on Figure 1.

It was found in the nonequilibri um supersomic nozzle flows CMach number $\sim 4$ ) due to the high rate of recombination pumping into radiative E-state $C R^{*} \geq 10^{20}$ (cms's and rapid VTdeactivation in absorbing $X$-state CTV not for much more than $T$, the partial population inversion Cdensity $\Delta N \geq$ $10^{13} \mathrm{~cm}^{3}$ is formed or ET $E, v^{\prime}=0,1 \rightarrow X, v^{\prime \prime}=14-$ $15, .$. in near IF spectrum $C \lambda=0.9 .1 .2 \mu m$. Exper imental varying of initial thermodynanic parameters To $2500-4000 \mathrm{~K}$. Po $=10-50$ atm, gas composition $C_{1}: A r=1: 0-1: s$ and nozzle flow regimes showed that the value of light gain a $\sim 10^{-3} \mathrm{~cm}$ is to be in good agreement with the theoretical predictions and sufficient to excite a laser oscillation. Gasdynamic quality of supersonic flow of recombining gas in the designed part of nozzles also was found to be a good enough.

The recombination scheme (15-(5) under flash photodissociation of $\mathrm{Br}$. was realized in photochemical pre-laser experiments [7], and 1 ight gain up to $5 \times 10^{-4} / 0 m$ ir 1 . O6 $\mu \mathrm{m}$ spectral region and specific laser energy up to $7 \mathrm{~J} / \mathrm{l}$ was achieved without any optimization. cPotentially specific laser energy can be increased by order of magnitude, and high efficiency up to 5...10\% can be anticipateds.

Recently the gasdynamic recombination studies for $C l$ and $3(B-X)$ systems have been started by LASERDOT (France) with the aim to obtain a chemically driven visible or mear IR GDL [8]. Unfortunately, the conditions of these experiments are unsufficient for 1 aser action, but they are promising in progress. 
It should be mentioned two unsuccessful attempts to demonstrate the RGDL when a preliminary phase of laser studies was ignored [9-10].

In experiments $[9]$ O $(\mathrm{B}-\mathrm{X})$ system was wrongly selected as 1 aser candidate, and the direct ${ }^{2}$ measurements in recombining gas flow in supersoric nozzles with a high quality mirrors has not resulted on the 1 aser effect. According with the numerical estimating, under these conditions the light gain was not more than of $10^{-6} \mathrm{~cm}$. The point is that EES of $O$, populating by 0 to atomic recombination, have strongly forbidden ET, ${ }^{2}$ thus 1 aser action could not be possible.

other experimentators [10] failed to demonstrate the FGDL directiy usirig the set-up of trivial heat pumped GDL. They rur the experiments simply replacing the co -containing mixture by the so, No and other dissociated gases but ${ }^{2}$ did not have any laser effect? Móreover, they have not been able to do any conclusions since they did not try to measure any characteristics of active medium in recombining gas flow. Such way without pre-laser investigations is not promising.

Besides the direct recombination 1 aser pumping scheme it is known the other principal possibilitiy to create a powerful visible GDL on ET Using a donor-acceptor schere based on E-E energy transfer. There are quite a number of fast chemical reactions, and atomic recombination reactions are among these, which have a high yield into metastable EES. Unfortunately, direct conversation of metastables's energy into laser radiation is difficult because its. ET into ground Es is spin-forbidden and has a very small cross-section. Such situation may be a case with the products of recombination of oxygen $-9(a) A$. nitroger - N CAD. Eut using this metastables as a EES energy donors, it can selects suitable acceptors. The criteria for choice of pare are ES excitation efriciency and radiative property of lasants. Here the halogers and halkogens are good lasing also due to partial inversion arising between the low energy levels of the radiative EES and the high errergy levels of the ground ES.

In conclusion, one should be stressed that all molecular candidates selected for RGDLs has been approbated in the lasers with optical pimping. If to corsider this laser operation process as complex of three sequential stages: - 13 high laser levels pumping, 23 stimulated radiative ET and 3 low laser levels depopulation experimentally two last ones has been successfully demonstrated, and $V-T$ ralaxation proved to be able "over-work" the molecular flow rate in CW lasing that comparable to analogous one for conditions of recontination pumping. This fact inspires our optimism.

\section{General Features and Perspective.}

\section{Expected RGDL performance}

RGDL on ET will be substantially differs from usual GDLs on VRT by following advantageous properties:

- more short wavelerigth of 1 asing $c \lambda=0,4-1,2 \mu m$;

- wide tuning of laser spectrum $(10-25 \%$;

- high stored energy per $1 \mathrm{~cm}^{2}$ of cross-section of a gas flow input to the optical cavity cup to $5-20 \mathrm{k} / \mathrm{cm} \mathrm{cm}^{2}$;

- high iaser enficiency (5. . 15\%.

For example, in the gas flow at cross-section $5 \times 50 \mathrm{~cm}^{2}$ it can be extracted the 1 aser output up to $50.2550 \mathrm{kH}$.

EGDL intracavity active media parameters will differ from GDLs too:

- more high pressure c200. . E00 Torrs;

- more high temperature (500. .5900 K);

- the lerght of inversion zone $\sim 10.50 \mathrm{~cm}$. 
Supposed RGDL applications

Because of its unique possibilities, the RGDL systems will have potential for all known high power laser applications in science, engineer 1 ng and technology.

In fact, for powerful laser technology applications, the co, and YAG lasers are most widely used now: the users of $\mathrm{CO}_{2}$ lasers take advantage of its high power output capability, and the users of YAG lasers focus on its high fiber transmission capability. The RGDLs will possess both important advantages simultaneously. While the power levels of RGDL is superior even to that of $\mathrm{CO}_{2}$
lasers in the same conditions, the oscillation wavelength lies near the minimum loss range of silica fibers. Therefore it is feasible to trarsmit and deliver $C W$ laser power through an optical fiber network system for a multi-station production combined with a robot and $X-Y$ material processing.

Decrease in diffraction divergance of RGDL's beam associated with the shorter wavelength results in the better focusibility $\left(\sim \lambda^{2}\right)$ which allows to increase the energy concentration on the taget as high as $100 \ldots 400$ times more than the CO, lasers of the same power level. Combining this effect with the 2 lesser reflectivity and greater absorptivity of metals $\left(\sim \lambda^{-3 / 2}\right)$, the RGDL may be expected to give more high quality of processing.

For laser energy production and wireless transmission applications the RGDL would operate in autonomous regime as they will not use an electric energy sources. Wide-range tunability of laser oscillation will open new possibilities both for loss-free communication and photochemical technology Claser-induced catalysis, isotope separation, etc.). Environment control will be possible as well.

At last, due to favour wavelength operation and high energy storage characteristics, an efficient repetitevely-pul sed cup to $10^{4}$ Hz) generator-amplifier can be expected to construct as alternative 1 aser driver system for fusion reactor research.

In spite of these rascinating properties nobody has tried to developed the RGDL for industrial application in mind probably because of some difficulties mentioned above. It motived us to take part in this challenge because it will be a discovery of new generation of $\mathrm{CW}$ high power short wavelength lasers and will have a significant impact in a number of key technologies.

\section{References.}

1/ Report of the APS Study Group. Rev. Mod. Phys. 59 (1987) S33-S68.

a, KOCHELAP V.A., PEKAR S.I. Theory of Spontaneous and St i mul ated Chemil umi nescence of Gases. - Kiev: Nauk. dumka (1986):

3/ LOSEV S.A. Gasdynamic Lasers. - Berlin: Springer-Verlag (1981).

/4/ BELOKRINI TSKY N.S. et al. Quant. Electron. 9 (1982) 298.

15/ KERNAZI TSKY L. A. et al. Chem. Phys. Lett. 116 (1985) 197.

6/ IZMAILOV I.A. et al. Arch. Combust. 8 (1988) 277.

7/ ANTIPENKO B.M. et al. Opt. \& Spectrosc. 57 (1984) 150.

8/ PRIGENT P. , BRUNET H. SPIE V.1031 (1988) 408.

(9/ VOLKOV A. Yu. et al. JETF 70 (1976) 2064.

10, FONTAINE B. , FORESTIER B. Quant. Electron. $3(1976) 897$. 